\title{
Perioral pressure ulcers in patients with COVID-19 requiring invasive mechanical ventilation
}

\author{
Aseel Sleiwah ${ }^{1}$ (1) $\cdot$ Ganeshkrishna Nair $^{2} \cdot$ Maleeha Mughal $^{1} \cdot$ Katie Lancaster $^{1} \cdot$ Imran Ahmad $^{2,3}$
}

Received: 20 May 2020 / Accepted: 1 September 2020 / Published online: 15 September 2020

(C) Springer-Verlag GmbH Germany, part of Springer Nature 2020

\begin{abstract}
Background Facial pressure ulcers are a rare yet significant complication. National Institute for Health and Care Excellence (NICE) guidelines recommend that patients should be risk-assessed for pressure ulcers and measures instated to prevent such complication. In this study, we report case series of perioral pressure ulcers developed following the use of two devices to secure endotracheal tubes in COVID-19 positive patients managed in the intensive care setting.

Methods A retrospective analysis was conducted on sixteen patients identified to have perioral pressure ulcers by using the institutional risk management system. Data parameters included patient demographics (age, gender, comorbidities, smoking history and body mass index (BMI)). Data collection included the indication of admission to ITU, duration of intubation, types of medical devices utilised to secure the endotracheal tube, requirement of vasopressor agents and renal replacement therapy, presence of other associated ulcers, duration of proning and mortality.

Results Sixteen patients developed different patterns of perioral pressure ulcers related to the use of two medical devices (Insight, AnchorFast). The mean age was 58.6 years. The average length of intubation was 18.8 days. Fourteen patients required proning, with an average duration of 5.2 days.

Conclusions The two devices utilised to secure endotracheal tubes are associated with unique patterns of facial pressure ulcers. Measures should be taken to assess the skin regularly and avoid utilising devices that are associated with a high risk of facial pressure ulcers. Awareness and training should be provided to prevent such significant complication.
\end{abstract}

Level of evidence: Level IV, risk/prognostic study.

Keywords Facial ulcers $\cdot$ Perioral ulcers $\cdot$ Pressure sores $\cdot$ Endotracheal intubation securing device

\section{Introduction}

The global health pandemic with coronavirus disease 2019 (COVID-19) caused a huge surge in admissions to critical care units worldwide for respiratory support and management of acute respiratory distress syndrome

Aseel Sleiwah

aseelnajeeb@yahoo.com

1 Department of Plastic Surgery, Guy's \& St. Thomas' Hospital, 3rd Floor, Lambeth Wing, Westminster Bridge, London SE1 7EH, UK

2 Department of Plastic Surgery, Guy's \& St. Thomas' Hospital, London, UK

3 King's College London, London, UK
(ARDS) $[1,2]$. Ventilating in a prone position is an important strategy to manage seriously ill patients with ARDS in an attempt to increase arterial oxygenation and reduce ventilation-perfusion mismatch [3-5]. Securing the airway is crucial to prevent accidental extubation or dislodgment of the tube into the right bronchus. Several medical devices have been designed to achieve this purpose [6]. We present a cohort of patients who had different patterns of perioral pressure ulcers related to two types of medical devices: endotracheal tube holder set (Insight Medical Products Ltd, Gloucestershire, UK) and AnchorFast device (Hollister, Libertyville, IL, USA). These devices were used to secure the endotracheal tube in COVID-19-positive patients treated in the intensive care unit as a consequence of severe ARDS (Figs. 1, 2). The face has an important 


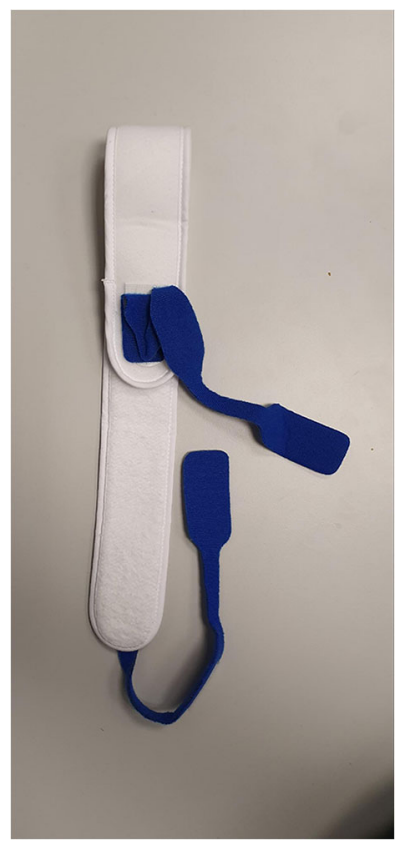

Fig. 1 Endotracheal tube-holding set

aesthetic function and scarring may result in distress and profound psychological impact. Hence, it is imperative to increase awareness among medical and nursing staff to this significant complication. As some healthcare systems are preparing for a potential second wave, protocols should be in place to prevent and minimise the risk of developing facial pressure sores [7, 8].

\section{Methods}

A retrospective chart review of patients identified to have perioral facial pressure ulcers in March and April

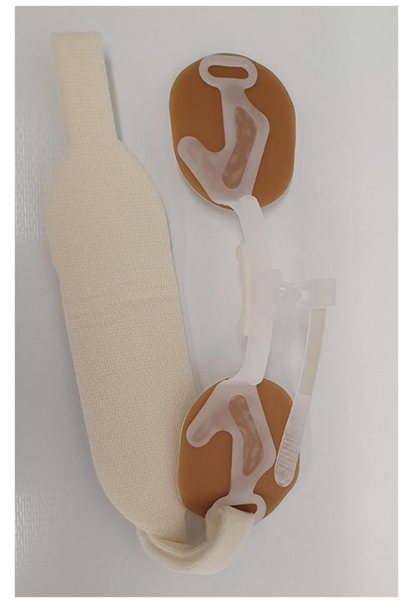

Fig. 2 AnchorFast device
2020 was conducted. All patients were identified through the hospital's incident reporting system. Data collection parameters included patient demographics (age, gender, comorbidities, smoking history, body mass index (BMI)). Also, the indication of admission to ITU, duration of intubation, types of medical devices utilised to secure the endotracheal tube, requirement of vasopressor agents and renal replacement therapy, presence of other associated ulcers, proning regimes, total days of proning and mortality were noted. Commonalities and variables were analysed. Proning procedures followed an agreed protocol with a minimum of six people and an anaesthetist to secure the artificial airway. Two wide sliding sheets were utilised with 4-6 pillows and half a crescent head jelly to protect the face. Pressure areas were assessed, and after turning the patient, they were placed in swimmer's position for 16-18 h.

\section{Results}

Sixteen patients were identified. The mean age was 58.6 years (range 40-77). Fourteen patients were males $(87 \%)$, while two were females (13\%). The average BMI was 28.8 (range 22-40.6). Their medical comorbidities and associated factors are shown in Table 1.

All patients were COVID-19 positive. Twelve patients were managed with proning for severe ARDS, while four patients did not require prone ventilation. The average duration of proning was 5.2 days (range 2-7 days). Fourteen patients had category two pressure injuries, one had category three, and one had unknown depth. The pressure ulcers around the lips follow a specific pattern depending on the type of device utilised to secure the endotracheal tube. Twelve patients had their airways secured with Insight Endotracheal Tube (ETT) Holder Set. These patients suffered injuries of the oral commissure (Fig. 3). An AnchorFast oral endotracheal tube fastener device was utilised to secure the tracheal tube in three patients; the pressure injury was related to the plastic part of the device on the upper lip and cheeks. Eight patients suffered other pressure ulcers (summarised in Table 1). One patient underwent extracorporeal membrane oxygenation (ECMO) for 14 days. Three patients sadly died; six patients were discharged from the hospital while the remainder seven are still being managed in the intensive care setting. 
Table 1 Characteristics of patients associated with perioral facial pressure ulcers

\begin{tabular}{|c|c|c|}
\hline \multicolumn{2}{|c|}{ Patient characteristics } & \multirow[t]{2}{*}{ Frequency (patient) } \\
\hline \multicolumn{2}{|l|}{ Ethnicity: } & \\
\hline & White & 8 \\
\hline & Black & 5 \\
\hline & Asian & 2 \\
\hline & Other & 1 \\
\hline \multicolumn{2}{|l|}{ Diabetes } & 7 \\
\hline \multicolumn{2}{|c|}{ Hypertension } & 7 \\
\hline \multicolumn{2}{|c|}{ Chronic kidney disease } & 2 \\
\hline \multicolumn{2}{|c|}{ Chronic obstructive lung disease } & 1 \\
\hline \multicolumn{2}{|l|}{ Smoking } & 0 \\
\hline \multirow[t]{2}{*}{ ETT size } & Size 9 & 12 \\
\hline & Size 8 & 4 \\
\hline \multicolumn{2}{|c|}{ Vasopressors } & 16 \\
\hline \multicolumn{2}{|c|}{ Duration of vasopressors use } & 6.3 days $(1-20)$ \\
\hline \multicolumn{2}{|c|}{ Duration of oral intubation } & 18.8 days $(11-34)$ \\
\hline \multicolumn{2}{|c|}{ Subsequent use of tracheostomy } & 10 \\
\hline \multicolumn{2}{|c|}{ Renal replacement therapy } & 6 \\
\hline \multicolumn{3}{|c|}{ Other pressure injuries identified } \\
\hline & Head & 5 \\
\hline & Foot & 2 \\
\hline & Knee & 2 \\
\hline & Chest & 1 \\
\hline & Sacrum & 1 \\
\hline & penis & 1 \\
\hline
\end{tabular}

\section{Discussion}

Acute respiratory distress syndrome (ARDS) is a relatively common complication of COVID-19 infection [2]. Placing patients in a prone position is an advocated treatment strategy to manage severe ARDS. Studies have shown improved survival when the oxygenation levels continue to fall despite the initial treatment measures taken [5]. The two main points to consider when placing patients in a prone position are firstly maintaining airway security and secondly monitoring pressure areas on bony prominences and sites related to medical devices and appliances. In our institution, designated proning teams were established to minimise the risks of such complications.

Securing of the tracheal tube is paramount to maintain both patient and staff safety [3,6]. It prevents the risk of accidental extubation which may lead to avoidable patient complications and mortality. This risk is especially high in the prone patient as more time is required to turn the position of the patient back to supine to re-establish the airway and ventilation. Besides, accidental extubation is a high aerosol-generating procedure (AGP). It, therefore, exposes the medical staff to risks of contracting the COVID-19 virus from the aerosols generated during mask ventilation and emergency reintubation. As a result, additional measures to establish and secure artificial airways were introduced during this pandemic [6].

Facial pressure ulcers are recognised risks from placing patients in a prone position. Girard et al. conducted a randomised control trial to examine pressure areas in 447 patients, comparing patients nursed in the supine position to those nursed in the prone position. They showed a higher incidence of pressure ulcers in prone patients and recommended that skin examination should be part of a daily management plan in the intensive care setting. Other risk factors identified in this study included male gender, age of greater than 60 years and body mass index of more than $28.5 \mathrm{~kg} / \mathrm{m}^{2}$ [9].

While there are many similarities among the patients in our cohort, the small sample size limits meaningful
Fig. 3 Picture showing scars from healed ulcers around the angles of the mouth
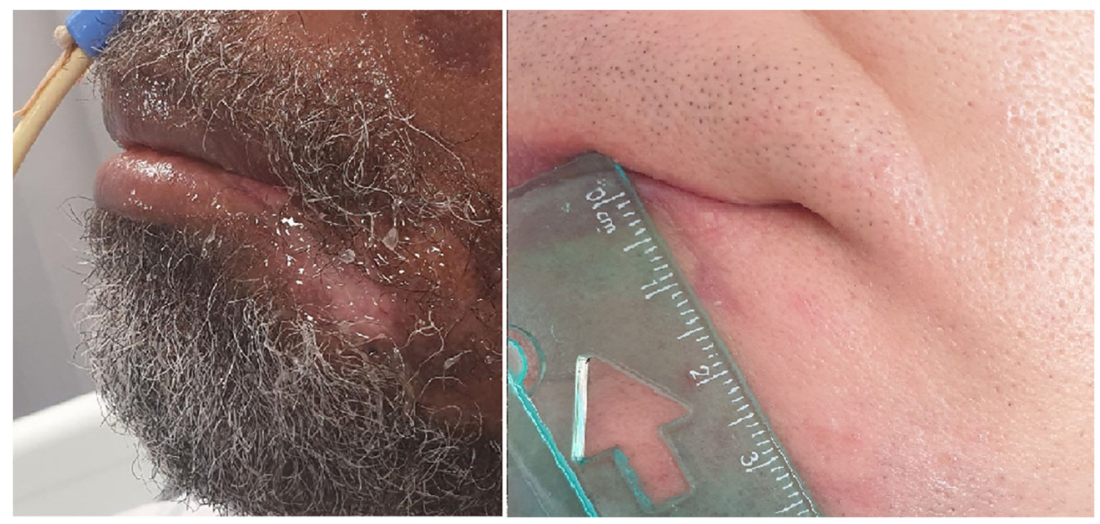
interpretation. More than half of them had medical comorbidities and all of our patients required inotropic support. They all had a prolonged course of oral intubation (average 18.8 days). Interestingly, none of them was a smoker, and the average BMI was only slightly elevated. While COVID-19 infection seems to affect the skin causing microvascular thrombotic injury [10], it is unclear if this significant factor is in our cohort of patients, especially that prominent skin necrosis elsewhere was not reported.

A systematic review and meta-analysis of 6 studies by Sud et al. combined data on 504 patients. It showed that 153 patients $(30.4 \%)$ had adverse events related to pressure areas; they concluded that patients in the prone position are associated with a higher incidence of pressure ulcers [11].

Many medical devices are utilised to secure artificial airways to reduce time and maximise efficiency. The two medical devices identified in our study were the AnchorFast device and ETT holder device (Insight). Pressure-related injuries from these devices follow a unique pattern. The pressure ulcers associated with the ETT-holding device (Insight) are mainly at the oral commissure. In contrast, the injuries sustained from the AnchorFast device are related to the plastic part and are distributed at the upper lip and cheeks. While four patients were in supine position, proning seems to be an essential risk factor, and a larger surface area is affected.

Appropriate use of securing devices is vital to prevent facial pressure ulcers. The ETT holder set (Insight) has two components: a blue Velcro-based tab and a softer, wider neckband. We observed that the blue tape causes pressure and friction-like effect on the angles of the mouth, causing the injuries mentioned above. Hence, it is imperative to correctly apply the device and select the right size for the patient (Fig. 4).

Few publications reported facial pressure ulcers associated with utilisation of AnchorFast device [12-14].

A study compared different types of commercial versus non-commercial devices to secure ETT in a simulated setting. It tested different types of devices measuring security of the ETT holder devices and pressure at different areas on the face using pressure sensors. They concluded that artificial airways exert more pressure on the face compared with non-commercial devices and that commercial devices allow more movement of the tube from one direction to another in a secure way. However, the study did not explore the devices in a prone position [6].

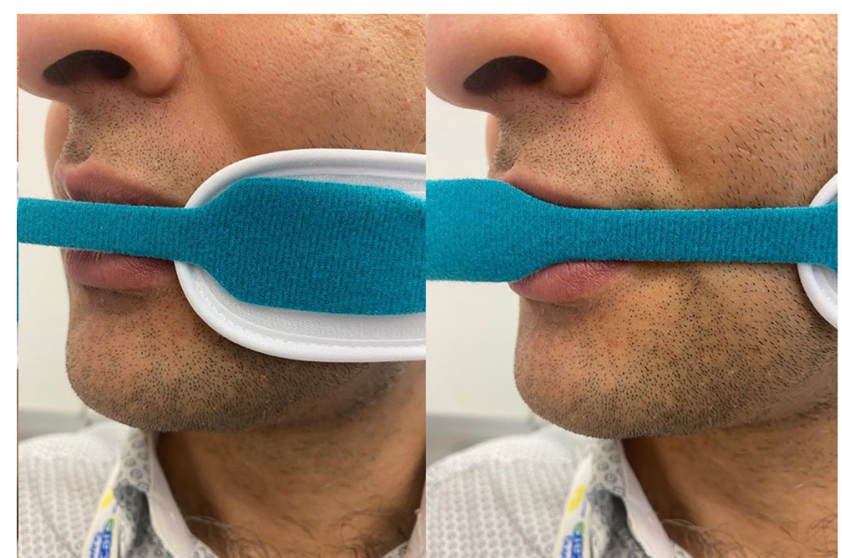

Fig. 4 Left, correct application of endotracheal tube-holding set; the blue tape should not be in direct contact with the lip commissure. Right, incorrect way of securing the endotracheal tube

The faculty of intensive care medicine guidance on proning recommends removing any AnchorFast device and securing the ETT with tape or tie [15].

Besides the mechanical effect of these devices, it seems that facial oedema from proning probably contributes to the pressure affect the skin [14].

Potential factors that may have led to the high incidence of facial pressure ulcers in our study include the rapid surge in numbers of intubated patients due to the COVID-19 pandemic coupled with increased demand on intensive care units. Also, lack of adequate staffing level and training, redeployment of nursing staff from other hospital areas and unfamiliarity with the new work environment may have led to failure to recognise the hazard of pressure ulcers from tracheal tube securing devices. The requirement to prone many patients at the same time mandated a team composed of health professionals ranging from surgeons, speech and language therapists, medical students and physiotherapist to help alleviate the pressure on clinical teams and reduce the manual workload [16]. As with many aspects of this new virus, a learning curve was soon evident as the reports highlighted the issue of facial pressure ulcers.

The treatment of the pressure ulcer around the mouth is mostly non-operative. Application of paraffin or chloramphenicol ointment helps to keep the wound moist. The face has a very good blood supply, and scar revisions are generally not indicated.

Potential preventative solutions reported in the literature include the use of a silicone foam (Mepliex; Mölnlycke) to decrease the shearing force between the skin and the device and use of a tie or tape to secure 
the ETT tube. Jackson et al. reported a case of facial ulcers resulted from securing the ETT with AnchorFast device. In their report, the patient remained prone for 5 days as she was unable to tolerate turns. She suffered severe pressure ulcers on the face. The authors suggested covering the face with silicone foam (Mölnlycke Health Care) and replacement of the AnchorFast device with a white fabric adhesive tape. Following their devised technique, they reported no new skin ulcers related to pressure on the face. Other authors followed similar protocols in a small cohort of patients [3]. In our unit, we are still investigating measures to prevent this significant complication, including removing the AnchorFast device and using Mepitel foam for high-risk patients.

In summary, perioral pressure ulcers are rare. However, the increased incidence of prone patients due to severe ARDS, unfamiliarity of clinical staff to the new work environment, potential lack of training and awareness, inappropriate use of medical devices to secure the ETT and failure to identify the hazard of developing facial pressure ulcers have resulted in an increased incidence of such complications in a cohort of COVID-19 patients.

Acknowledgments We would like to thank Roberson Filipini and tissue viability team for their role in patient identification and clinical input.

Availability of data and material I confirm that all data and materials support the published claims and comply with the journal standards.

\section{Compliance with ethical standards}

Code availability Not applicable.

Funding None.

Conflict of interest Aseel Sleiwah, Ganeshkrishna Nair, Maleeha Mughal, Katie Lancaster and Imran Ahmad declare that they have no conflict of interest.

Ethics approval All procedures performed in studies involving human participants were in accordance with the ethical standards of the institutional and/or national research committee and with the 1964 Helsinki Declaration and its later amendments or comparable ethical standards. This observational project was conducted following a departmental registration and approval.

Consent to participate Informed consents was obtained from all patients and next of kin (for deceased patients) who participated in this study.
Consent for publication Informed consent was obtained for the purpose of publication from all individual participants and next of kin (for deceased patients) included in the study.

\section{References}

1. Wang C, Horby PW, Hayden FG, Gao GF (2020) A novel coronavirus outbreak of global health concern [published correction appears in Lancet. 2020 Jan 29]. Lancet. 395(10223):470-473

2. Wang D, Hu B, Hu C et al (2020) Clinical characteristics of 138 hospitalized patients with 2019 novel coronavirus-infected pneumonia in Wuhan, China. JAMA 323(11):1061-1069

3. Meng L, Qiu H, Wan L et al (2020) Intubation and ventilation amid the COVID-19 outbreak: Wuhan's experience [published online ahead of print, 2020 Mar 26]. Anesthesiology. https://doi.org/10. 1097/ALN.0000000000003296

4. Fan E, Del Sorbo L, Goligher EC et al (2017) An official American Thoracic Society/European Society of Intensive Care Medicine/ Society of Critical Care Medicine Clinical Practice Guideline: mechanical ventilation in adult patients with acute respiratory distress syndrome [published correction appears in Am J Respir Crit Care Med. 2017 Jun 1;195(11):1540]. Am J Respir Crit Care Med 195(9):1253-1263

5. Guérin C, Reignier J, Richard JC, Beuret P, Gacouin A, Boulain T, Mercier E, Badet M, Mercat A, Baudin O, Clavel M, Chatellier D, Jaber S, Rosselli S, Mancebo J, Sirodot M, Hilbert G, Bengler C, Richecoeur J, Gainnier M, Bayle F, Bourdin G, Leray V, Girard R, Baboi L, Ayzac L (2013) Prone positioning in severe acute respiratory distress syndrome. N Engl J Med 368(23):2159-2168

6. Fisher DF, Chenelle CT, Marchese AD, Kratohvil JP, Kacmarek RM (2014) Comparison of commercial and noncommercial endotracheal tube-securing devices. Respir Care 59(9):1315-1323

7. Leung K, Wu JT, Liu D, Leung GM (2020) First-wave COVID-19 transmissibility and severity in China outside Hubei after control measures, and second-wave scenario planning: a modelling impact assessment. Lancet. 395(10233):1382-1393

8. Xu S, Li Y (2020) Beware of the second wave of COVID-19. Lancet. 395(10233):1321-1322

9. Girard R, Baboi L, Ayzac L, Richard JC, Guérin C (2014) Proseva trial group. The impact of patient positioning on pressure ulcers in patients with severe ARDS: results from a multicentre randomised controlled trial on prone positioning. Intensive Care Med 40(3): 397-403

10. Magro C, Mulvey JJ, Berlin D et al (2020) Complement associated microvascular injury and thrombosis in the pathogenesis of severe COVID-19 infection: a report of five cases [published online ahead of print, 2020 Apr 15]. Transl Res S1931-5244(20):30070-0

11. Sud S, Sud M, Friedrich JO, Adhikari NK (2008) Effect of mechanical ventilation in the prone position on clinical outcomes in patients with acute hypoxemic respiratory failure: a systematic review and meta-analysis. CMAJ. 178(9):1153-1161

12. Jackson ME, Verano JX, Fry JE, Rodriguez AP, Russian C (2012) Skin preparation process for the prevention of skin breakdown in patients who are intubated and treated with RotoProne. Respir Care 57(2):311-314

13. Kim RS, Mullins K (2016) Preventing facial pressure ulcers in acute respiratory distress syndrome (ARDS). J Wound Ostomy Continence Nurs 43(4):427-429 
14. Gomaa D, Branson RD (2015) Endotracheal tube holders and the prone position: a cause for concern. Respir Care 60(2):e41-e42

15. Guidance For: Prone positioning in adult critical care. The faculty of intensive care medicine. Accessed online from https://www. ficm.ac.uk/sites/default/files/prone_position_in_adult_critical_ care_2019.pdf. Accessed 15 May 2020
16. Mayer HF, Persichetti P (2020) Plastic surgery during the COVID19 pandemic times. Eur J Plast Surg 43(3):361-362. https://doi.org/ $10.1007 / \mathrm{s} 00238-020-01685-1$

Publisher's note Springer Nature remains neutral with regard to jurisdictional claims in published maps and institutional affiliations. 